\title{
Vital Signs: Trends in HIV Diagnoses, Risk Behaviors, and Prevention Among Persons Who Inject Drugs — United States
}

\author{
Cyprian Wejnert, $\mathrm{PhD}^{1}$; Kristen L. Hess, $\mathrm{PhD}^{1}$; H. Irene Hall, $\mathrm{PhD}^{1}$; Michelle Van Handel, $\mathrm{MPH}^{1}$; Demorah Hayes, MA ${ }^{1}$; Paul Fulton, Jr. ${ }^{1}$; \\ Qian An, $\mathrm{PhD}^{1}$; Linda J. Koenig, $\mathrm{PhD}^{1}$; Joseph Prejean, $\mathrm{PhD}^{1}$; Linda A. Valleroy, $\mathrm{PhD}^{1}$
}

On November 29, 2016, this report was posted as an MMWR Early Release on the MMWR website (http://www.cdc.gov/mmwr).

\section{Abstract}

Background: Persons who inject drugs (PWID) are at increased risk for poor health outcomes and bloodborne infections, including human immunodeficiency virus (HIV), hepatitis $\mathrm{C}$ virus and hepatitis B virus infections. Although substantial progress has been made in reducing HIV infections among PWID, recent changes in drug use could challenge this success.

Methods: CDC used National HIV Surveillance System data to analyze trends in HIV diagnoses. Further, National HIV Behavioral Surveillance interviews of PWID in 22 cities were analyzed to describe risk behaviors and use of prevention services among all PWID and among PWID who first injected drugs during the 5 years before their interview (new PWID).

Results: During 2008-2014, HIV diagnoses among PWID declined in urban and nonurban areas, but have leveled off in recent years. Among PWID in 22 cities, during 2005-2015, syringe sharing decreased by 34\% among blacks/African Americans (blacks) and by 12\% among Hispanics/Latinos (Hispanics), but remained unchanged among whites. The racial composition of new PWID changed during 2005-2015: the percentage who were black decreased from 38\% to 19\%, the percentage who were white increased from $38 \%$ to $54 \%$, and the percentage who were Hispanic remained stable. Among new PWID interviewed in 2015, whites engaged in riskier injection behaviors than blacks.

Conclusions: Decreases in HIV diagnoses among PWID indicate success in HIV prevention. However, emerging behavioral and demographic trends could reverse this success.

Implications for public health practice: Access to comprehensive prevention services is essential for all PWID. Syringe services programs reduce syringe sharing and can help PWID access prevention and treatment services for HIV and other bloodborne diseases, such as hepatitis $\mathrm{C}$ and hepatitis $\mathrm{B}$.

\section{Introduction}

Persons who inject drugs (PWID) are at higher risk for human immunodeficiency virus (HIV) infection. Although acquired immune deficiency syndrome (AIDS) diagnoses among PWID have decreased approximately $90 \%$ since their peak in 1993 (1), and only an estimated $0.3 \%$ of the U.S. population has injected drugs in the past 12 months (2), approximately $9 \%$ of HIV infections diagnosed in recent years are among PWID (3). Although HIV diagnoses among PWID have decreased over time (3), recent increases in acute hepatitis $\mathrm{C}$ virus (HCV) infections (4), which are frequently associated with injection drug use, suggest this progress could be negatively affected by increases in opioid (5) and heroin (6) use. The U.S. National HIV/AIDS Strategy* identifies PWID

\footnotetext{
*https://www.aids.gov/federal-resources/national-hiv-aids-strategy/nhas-update.pdf.
}

as a priority population for HIV prevention. Surveillance data were analyzed to determine recent trends in annual HIV diagnoses and characteristics and behaviors of PWID to better understand emerging opportunities and challenges in HIV prevention among PWID.

\section{Methods}

CDC's National HIV Surveillance System data reported through June 2016 from 50 states and the District of Columbia were used to obtain the number of PWID with HIV diagnosed ${ }^{\dagger}$ during 2008-2014. Data were statistically adjusted for missing transmission categories by multiple imputation (7). National HIV Behavioral Surveillance (8) (NHBS) data include PWID who injected drugs during the 12 months before their NHBS interview in 22 U.S. cities

\footnotetext{
$\dagger$ Includes persons with HIV infection with a history of ever injecting drugs, including men who ever had sex with men and injected drugs.
} 
during 2005-2015. ${ }^{\S}$ Among all PWID, trend analyses $\$$ of receipt of syringes** and syringe sharing ${ }^{\dagger \dagger}$ were conducted to understand changes in HIV-associated injection behaviors. Additional analyses were limited to PWID who injected drugs for the first time during the 5 years before interview $\$ \$$ (new PWID) for 1) trends since 2005 in racial/ethnic composition's of new PWID, and 2) comparisons of demographic characteristics, HIV and HCV testing, injection behaviors, and $\mathrm{HIV}^{* * *}$ and self-reported $\mathrm{HCV}$ prevalence, by race/ ethnicity ${ }^{\dagger \dagger}$ among new PWID interviewed in 2015.

\section{Results}

During 2008-2014, HIV diagnoses among PWID in the United States decreased by $48 \%$ (from 6,604 to 3,461). During this same period, diagnoses among black/African American (black) and Hispanic/Latino (Hispanic) PWID decreased by approximately $50 \%$ in both urban (from 2,452 to 950 among blacks; from 1,185 to 639 among Hispanics) and nonurban (from 523 to 222 among blacks; from 197 to 95 among

\footnotetext{
$\$$ National HIV Behavioral Surveillance data include PWID recruited using respondent-driven sampling in 22 U.S. cities during 2005-2006, 2009, 2012, and 2015. The target sample size in each city each year was 500 PWID. The number of cities included each year varied; 2005: 22 cities; 2009, 2012, and 2015: 19 cities. The following cities were included in all years: Atlanta, Georgia; Baltimore, Maryland; Boston, Massachusetts; Chicago, Illinois; Dallas, Texas; Denver, Colorado; Detroit, Michigan; Houston, Texas; Los Angeles, California; Miami, Florida; Nassau-Suffolk, New York; New York City, New York; Newark, New Jersey; Philadelphia, Pennsylvania; San Diego, California; San Francisco, California; Seattle, Washington. Additional cities were included as follows: in 2005, Fort Lauderdale, Florida; Las Vegas, Nevada; Norfolk-Portsmouth, Virginia; St. Louis, Missouri; in 2009, 2012, 2015, New Orleans, Louisiana; Washington, D.C.

Separate Poisson regression models with generalized estimating equations (GEE) clustered on recruitment chain were used to examine trends overall and stratified by race/ethnicity. Interview year was included in the models as an ordinal variable to examine changes over time. Models were adjusted for continuous age to account for changing demographics over time. A single model utilizing interactions by race yielded similar results.

** A person was considered to have received a syringe from a syringe services program (SSP) if he or she reported receiving a sterile syringe or needle, at least once, from an SSP or syringe/needle exchange program during the 12 months before interview. A person was considered to have received syringes only from sterile sources if he or she reported receiving syringes from at least one of the following: SSP, pharmacy, or healthcare provider and NOT any other source during the 12 months before interview.

许 A person was considered to have shared a syringe during the 12 months before interview if he or she reported using a syringe to inject drugs that had previously been used for drug injection by someone else.

$\$ \$$ Calculated based on age at first injection and year of interview.

99 To assess trends in the racial composition of new PWID, we defined three dummy variables representing blacks/African Americans (blacks), Hispanics/Latinos (Hispanics), and whites, and conducted Poisson regression models with GEE clustered on recruitment chain. For each racial/ethnic group, the corresponding dummy variable was included in the model as a dependent variable, year as an ordinal variable, and continuous age as a covariate.

*** Determined by HIV test administered immediately after the interview.

t†† P-values comparing blacks to whites and Hispanics to whites were calculated using GEE regression clustered on recruitment chain, with race/ethnicity as the covariate. All models were adjusted for continuous age, except for analysis of age and age at first injection.
}

Hispanics) areas, $\$ \$ \$ \$$ with a slower decrease observed in the more recent years (Figure 1). Diagnoses among white PWID in urban areas decreased by $28 \%$ (from 1,350 to 977 ) during 2008-2012, but remained stable past 2012. In nonurban areas, diagnoses among white PWID decreased by $28 \%$ (from 481 to 345 ) during 2008-2010, but remained stable during 2010-2014. The majority of HIV diagnoses (79\%) among PWID occurred in urban areas.

NHBS data999 indicate that, although the percentage of PWID in 22 cities who received syringes from a syringe services program (SSP)**** (9) increased during 2005-2015 for all racial/ethnic groups $(\mathrm{p}<0.001)$, less than one third of PWID received all their syringes from sterile sources (Figure 2). During 2005-2015, the percentage of black PWID who received all their syringes from sterile sources increased by $48 \%(\mathrm{p}<0.001)$. During the same period, the percentages of Hispanic PWID and white PWID who received all their syringes from sterile sources did not change (Hispanics, $\mathrm{p}=0.16$, whites, $\mathrm{p}=0.72$ ). Hispanic PWID had the largest percentage of persons receiving all their syringes from sterile sources in each year. In 2015 , overall $25 \%$ of PWID interviewed received all their syringes from sterile sources (blacks $=28 \%$; Hispanics $=29 \%$; whites $=22 \%$ ) (Figure 2).

During 2005-2015, the percentages of black and Hispanic PWID in 22 cities who shared syringes decreased (blacks, $\mathrm{p}<0.001$; Hispanics, $\mathrm{p}=0.005$ ) (Figure 2). The largest decrease, 34\%, was among black PWID, followed by Hispanic PWID (12\%). During 2005-2015, the percentage of white PWID who shared syringes did not change $(\mathrm{p}=0.14)$. In 2015, the highest percentage of PWID interviewed who shared syringes were white $(43 \%)$, followed by Hispanic $(33 \%)$ and black $(21 \%)$. Across all years, $31 \%$ of PWID who received at least one syringe from SSPs had shared syringes, compared with $38 \%$ who had not received any syringes from SSP. Among PWID who received all their syringes from sterile sources, ${ }^{\dagger \dagger \dagger \dagger}$ $13 \%$ had shared syringes in the past year; $41 \%$ of PWID who did not receive all their syringes from sterile sources ${ }^{\$ \$ \$ \$}$ had shared syringes in the previous year.

\footnotetext{
$\$ \$ \$$ Urban areas include metropolitan statistical areas with populations of $\geq 500,000$ persons; areas with populations $<500,000$ persons were considered nonurban.

999 NHBS data are limited to PWID in urban areas who likely have greater access to SSPs and other resources than PWID outside of urban centers.

**** SSPs and syringe/needle exchange programs allow PWID to exchange used syringes or needles for new, sterile syringes. SSPs provide comprehensive services such as HIV or HCV testing, linkage to care, and medication-assisted treatment. NHBS does not collect information on what services are offered at SSPs.

itt† Sterile sources include SSPs, pharmacies, and health care providers.

$\$ \$ \$ \$$ Includes PWID who only received syringes from nonsterile sources and PWID who received syringes from both sterile and nonsterile sources. Among PWID who only received syringes from nonsterile sources, $41 \%$ shared syringes; among PWID who received syringes from both sterile and nonsterile sources $42 \%$ shared syringes in the past 12 months.
} 
During 2005-2015, the racial/ethnic distribution of new PWID changed (Figure 3). The percentage of new PWID who were black decreased by $51 \%$, from $38 \%$ in 2005 to $19 \%$ in 2015 ( $\mathrm{p}<0.001)$. The percentage who were Hispanic remained stable at approximately $21 \%(\mathrm{p}=0.72)$. The percentage who were white increased by $40 \%$ from $38 \%$ in 2005 to $54 \%$ in $2015(\mathrm{p}=0.002)$.

In $2015,54 \%$ of new PWID were white, $21 \%$ were Hispanic, and 19\% were black (Table). (In 2015, among PWID who had been injecting for $\geq 5$ years, $37 \%$ were white, $18 \%$ were Hispanic, and $40 \%$ were black.) Overall, $48 \%$ of new PWID were aged 18-29 years; however, variations by race/ethnicity were observed: $21 \%$ of black, $49 \%$ of Hispanic, and $56 \%$ of white new PWID were aged 18-29 years. Syringe sharing was most commonly reported among whites (46\%), followed by Hispanics (32\%) and blacks (28\%). Regardless of race/ethnicity, most new PWID injected at least one time per day $(83 \%)$ and primarily injected heroin $(27 \%)$ or heroin and other drugs (61\%) during the 12 months before their interview. Among new PWID, prescription opioid misuse during the past 12 months was most common among whites (27\% injection; $46 \%$ noninjection), followed by Hispanics (21\% injection; $37 \%$ noninjection) and blacks (10\% injection; $30 \%$ noninjection). HIV testing in the 12 months before the interview was most commonly reported among blacks (65\%), followed by Hispanics (58\%) and whites (57\%). HIV prevalence was highest among blacks (9\%), followed by Hispanics (4\%) and whites $(2 \%)$. Having ever been tested for HCV was most common among whites (72\%), followed by blacks $(69 \%)$ and Hispanics (64\%). Self-reported HCV prevalence, among new PWID who had previously been tested, was highest among whites (22\%), followed by Hispanics (19\%) and blacks (13\%). New PWID who received syringes from SSPs were more likely to dispose of all their syringes safely (37\%) than those who had not received syringes from SSPs (7\%).

\section{Conclusions and Comments}

Trends in protective behavior might explain why HIV diagnoses have declined more among black PWID than among white PWID. Fewer blacks are injecting drugs now than before (10) and the percentage of new PWID who are black decreased by $51 \%$ during 2005-2015. Further, during that time, the percentage of black PWID who shared syringes decreased by 34\%. During 2005-2015, the percentage of new white PWID increased by $40 \%$; syringe sharing among white PWID remained high, at approximately $43 \%$.

Black PWID currently account for $<20 \%$ of new PWID. This decrease might be partially explained by changes in social norms regarding drug injection in black communities (10), as well as increases in injection drug use among whites (11).
FIGURE 1. Diagnoses of human immunodeficiency virus (HIV) infection among persons who inject drugs - National HIV Surveillance System, ${ }^{*}$ United States, 2008-2014

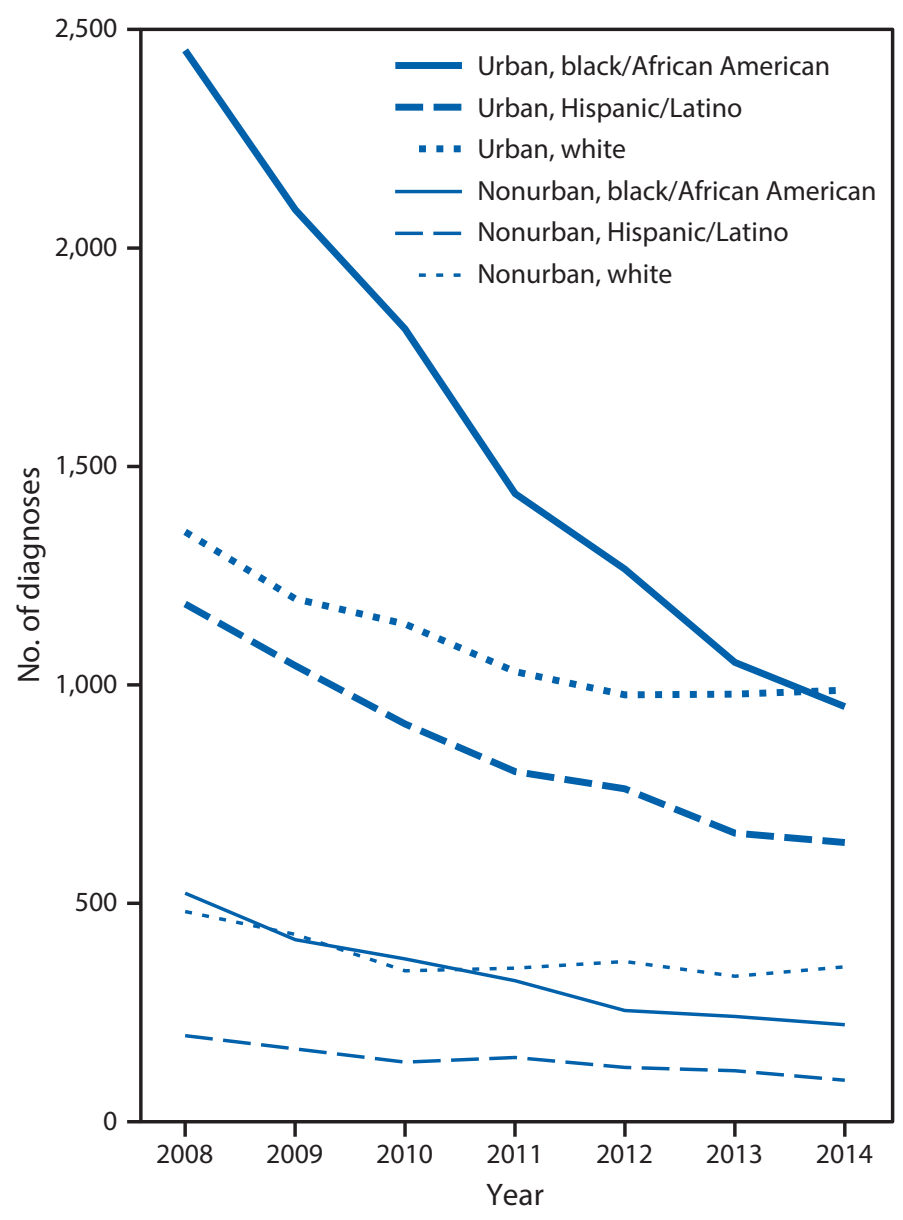

${ }^{*}$ http://www.cdc.gov/hiv/statistics/surveillance/index.html.

Black PWID tend to be older, and fewer report sharing syringes than do Hispanics or whites (12). Encouraging trends in use of SSPs and receipt of all syringes from sterile sources contribute to reducing the risk for HIV among black PWID. However, blacks remain at increased risk for HIV because of the high prevalence of HIV in their communities (3).

HIV diagnoses also decreased among Hispanic PWID. Although the data do not suggest that fewer Hispanics inject drugs now than previously, behavioral surveillance data suggest a trend of reduced syringe sharing, which is potentially related to increased access to SSPs and relatively high, steady rates of receiving all syringes from sterile sources.

Although HIV diagnoses among white PWID have decreased since 2008, recent trends suggest heroin use and injection drug use among whites are increasing $(10,11)$ and, coupled with high rates of syringe sharing, might challenge the decades of progress in HIV prevention among PWID. For the first time, in 2014, a larger number of white PWID received an HIV 
FIGURE 2. Trends in receipt of syringes and syringe sharing in the past 12 months among persons who inject drugs, by year - National HIV Behavioral Surveillance, ${ }^{*}$ selected cities, United States, 2005-2015

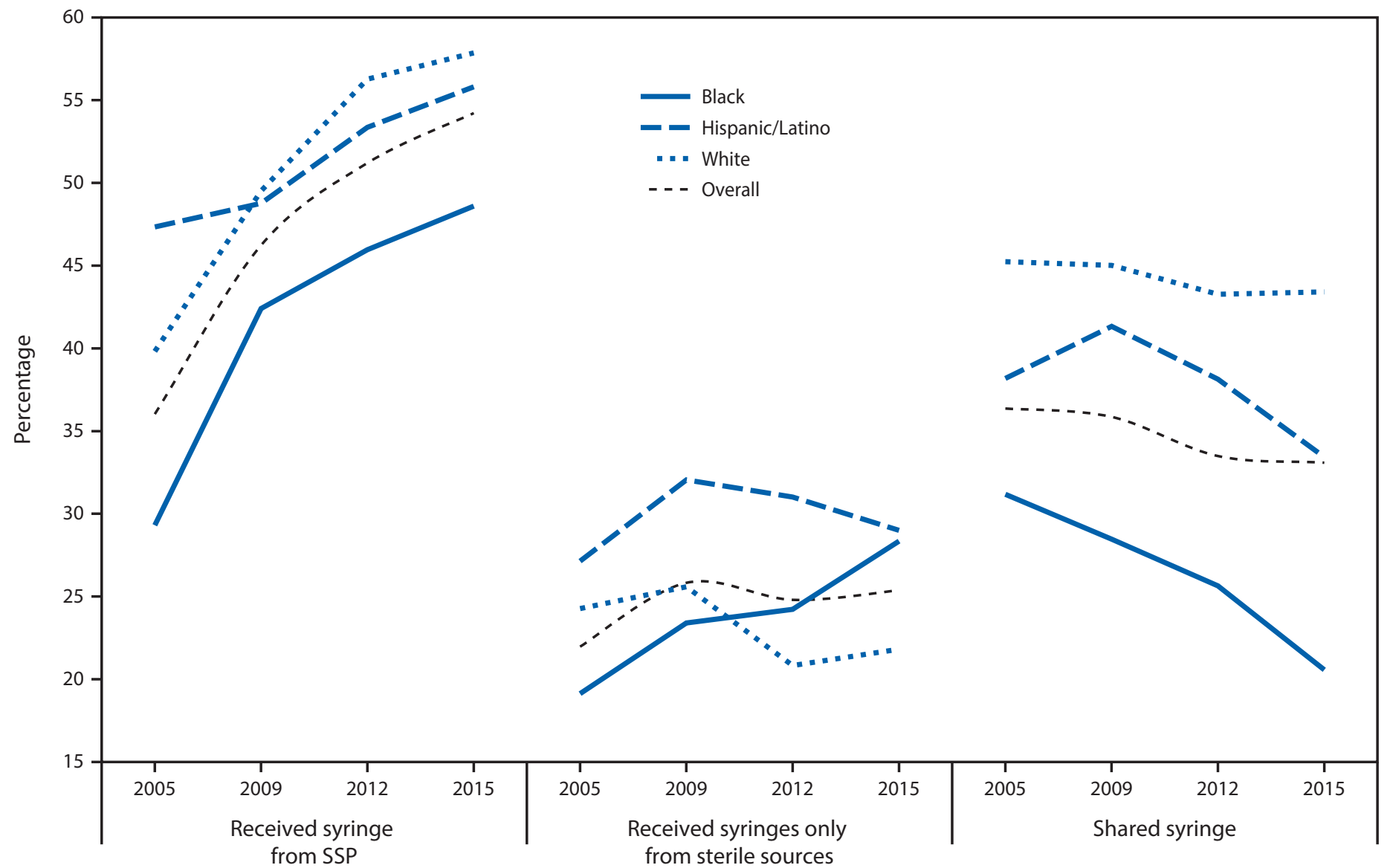

Abbreviations: $\mathrm{HIV}=$ human immunodeficiency virus; SSP = syringe services program.

* http://www.cdc.gov/hiv/statistics/systems/nhbs/. National HIV Behavioral Surveillance data include persons who inject drugs (PWID) who injected drugs during the past 12 months before being interviewed. PWID were recruited using respondent-driven sampling in 22 U.S. cities during 2005-2006, 2009, 2012, and 2015. The target sample size in each city each year was 500 PWID. The number of cities included each year varied: 2005: 22 cities; 2009, 2012, and 2015: 19 cities. The following cities were included in all years: Atlanta, Georgia; Baltimore, Maryland; Boston, Massachusetts; Chicago, Illinois; Dallas, Texas; Denver, Colorado; Detroit, Michigan; Houston, Texas; Los Angeles, California; Miami, Florida; Nassau-Suffolk, New York; New York City, New York; Newark, New Jersey; Philadelphia, Pennsylvania; San Diego, California; San Francisco, California; Seattle, Washington. Additional cities were included as follows: in 2005: Fort Lauderdale, Florida; Las Vegas, Nevada; NorfolkPortsmouth, Virginia; St. Louis, Missouri; in 2009, 2012, 2015: New Orleans, Louisiana; Washington, D.C.

diagnosis than PWID from any other racial/ethnic population in the United States. This analysis indicates that white PWID, who tend to begin injecting at younger ages than blacks or Hispanics (12), accounted for $>50 \%$ of new PWID in 2015. Further, although white PWID in 22 cities reported increases in receipt of sterile syringes from SSPs similar to increases reported among blacks and Hispanics, they were least likely to receive all their syringes from sterile sources and most likely to have shared syringes. Rural areas, which are predominately white, include some of the most vulnerable populations for injection drug use and injection drug use-related HIV outbreaks and might have the greatest unmet need for risk reduction services provided by SSPs $(9,13,14)$.
SSPs reduce syringe sharing and are widely considered to be an effective means for reducing HIV transmission.9999 Reducing syringe sharing through improved access to SSPs is a critical component of HIV prevention among PWID. Although access to syringes from SSPs has increased, the supply of sterile syringes available to most PWID is likely to be insufficient to meet their needs, and barriers remain to accessing SSPs, including lack of SSPs in rural areas (9) and absence of legal support in many states (15).***** NHBS data indicate that $83 \%$ of new PWID interviewed injected one or more times per day; comparing estimates of PWID population size (2) with the estimated number of syringes distributed by SSPs in 2013

999976 FR 10038 (https://www.federalregister.gov/a/2011-3990).

***** Only 17 states and Washington, D.C. have laws that explicitly authorize SSPs (http://www.lawatlas.org). 
FIGURE 3. Race/ethnicity of persons who reported injecting drugs for the first time during the 5 years before interview - National HIV Behavioral Surveillance, ${ }^{*}$ selected cities, United States, 2005-2015 ${ }^{\dagger}$

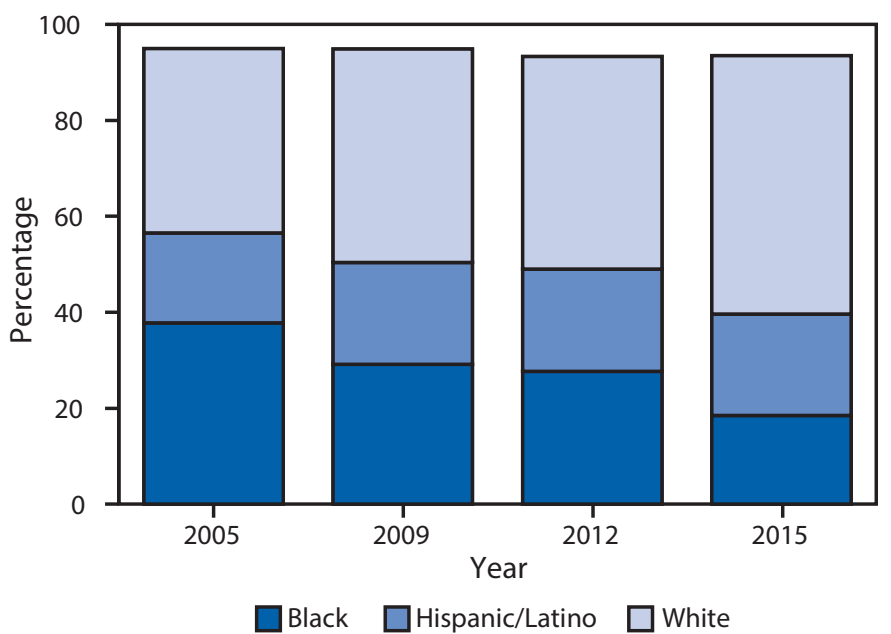

Abbreviation: HIV = human immunodeficiency virus.

* http://www.cdc.gov/hiv/statistics/systems/nhbs/. National HIV Behavioral Surveillance data include persons who inject drugs (PWID) who injected drugs during the 12 months before being interviewed. Graph data are limited to PWID who injected drugs for the first time during the past 5 years before being interviewed (considered"new" PWIDs). PWID were recruited using respondentdriven sampling in 22 U.S. cities during 2005-2006, 2009, 2012, and 2015. The target sample size in each city each year was 500 PWID. The number of cities included each year varied: 2005: 22 cities; 2009, 2012, and 2015: 19 cities. The following cities were included in all years: Atlanta, Georgia; Baltimore, Maryland; Boston, Massachusetts; Chicago, Illinois; Dallas, Texas; Denver, Colorado; Detroit, Michigan; Houston, Texas; Los Angeles, California; Miami, Florida; Nassau-Suffolk, New York; New York City, New York; Newark, New Jersey; Philadelphia, Pennsylvania; San Diego, California; San Francisco, California; Seattle, Washington. Additional cities were included as follows: in 2005: Fort Lauderdale, Florida; Las Vegas, Nevada; Norfolk-Portsmouth, Virginia; St. Louis, Missouri; in 2009, 2012, 2015: New Orleans, Louisiana; Washington, D.C.

+ Percentages for "other, multiple races" not included; therefore, bars do not add to $100 \%$.

(9) and the low percentage (25\%) of PWID in 22 cities who received all their syringes from sterile sources suggests that the U.S. syringe supply falls short. In addition to providing PWID with sterile syringes and equipment, SSPs serve as a bridge to access condoms; risk-reduction education; testing for HIV and HCV; referrals to health services, such as treatment for HIV, HCV, or substance use disorder, including medicationassisted treatment (e.g., with methadone or buprenorphine); pre-exposure prophylaxis (i.e., PrEP); and safe syringe disposal (9). SSPs are an effective tool for reducing substance misuse and risk for HIV infection. Recent changes in federal law ${ }^{\dagger \dagger \dagger \dagger \dagger}$ afford an opportunity to improve provision of comprehensive prevention services to all PWID through SSPs. It is important for jurisdictions to understand patterns of substance use in their communities, assess their SSP needs, and ensure services are provided to PWID (13).

\section{Key Points}

- Persons who inject drugs (PWID) are at increased risk for human immunodeficiency virus (HIV), hepatitis C virus $(\mathrm{HCV})$, hepatitis $\mathrm{B}$ virus, and other negative health outcomes. In 2014, 9\% of HIV diagnoses were among PWID. Although substantial progress has been made in reducing HIV infections among PWID, recent changes in drug use could challenge this success.

- HIV diagnoses among black/African American (black) and Hispanic/Latino PWID decreased about 50\% during 2008-2014. Blacks now make up 19\% of new PWID in 22 cities, down from $38 \%$ in 2005 . Syringe sharing decreased among black and Hispanic/Latino PWID in 22 cities.

- Diagnoses among urban white PWID decreased 28\%, but the decline stopped in 2012. Whites continue to have the highest rate of syringe sharing and now make up over $50 \%$ of new PWID.

- Among PWID who received all their syringes from sterile sources in the past year, $13 \%$ shared syringes; $41 \%$ of PWID who did not receive all their syringes from sterile sources shared syringes.

- Strategies that reduce HIV infections and syringe sharing among PWID should be used to meet emerging challenges in changing PWID populations. Syringe services programs reduce syringe sharing and serve as a bridge to other health services including HIV and $\mathrm{HCV}$ diagnosis and treatment and medication-assisted treatment for substance use disorder.

- Additional information is available at http://www.cdc. gov/vitalsigns.

The findings in this report are subject to at least three limitations. First, National HIV Surveillance System data are subject to reporting delays, and not all cases might have been reported at this time. This report presents trends in HIV diagnoses through 2014; preliminary 2015 data are not reliable for trend analysis (3). Second, NHBS data are limited to PWID recruited in large U.S. cities with high HIV prevalences using respondent-driven sampling and might not be generalizable to all PWID; recruitment in urban areas might not reflect the demographics and syringe use patterns of PWID living in rural areas. Finally, behavioral data and HCV prevalence are self-reported and subject to social desirability bias.

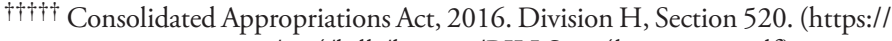
www.congress.gov/114/bills/hr2029/BILLS-114hr2029enr.pdf). 
TABLE. Characteristics and risk behaviors among persons who inject drugs (PWID) whose first injection was during 2010-2015 (new PWID), by race/ethnicity - National HIV Behavioral Surveillance, ${ }^{*} 19$ cities, ${ }^{\dagger}$ United States, 2015

\begin{tabular}{|c|c|c|c|c|c|c|}
\hline \multirow[b]{3}{*}{ Characteristic/Risk behavior } & \multicolumn{5}{|c|}{ New PWID } & \multirow{3}{*}{$\begin{array}{c}\text { Total }^{\S} \\
\text { No. (\%) }\end{array}$} \\
\hline & \multicolumn{2}{|c|}{ Black (19\%) } & \multicolumn{2}{|c|}{ Hispanic/Latino (21\%) } & \multirow{2}{*}{$\begin{array}{c}\text { White (54\%) } \\
\text { No. (\%) }\end{array}$} & \\
\hline & No. (\%) & $\begin{array}{c}p \text {-value } \\
\text { versus white }\end{array}$ & No. (\%) & $\begin{array}{c}\text { p-value } \\
\text { versus white }\end{array}$ & & \\
\hline \multicolumn{7}{|l|}{ Sex/Gender } \\
\hline Male & $228(65.9)$ & 0.198 & $270(69.2)$ & 0.049 & $640(64.1)$ & $1,212(65.2)$ \\
\hline Female & $113(32.7)$ & 0.112 & $119(30.5)$ & 0.066 & $355(35.6)$ & $635(34.2)$ \\
\hline Transgender & $5(1.4)$ & 一** & $1(0.3)$ & - ** & $3(0.3)$ & $11(0.6)$ \\
\hline \multicolumn{7}{|l|}{ Age group (yrs) } \\
\hline $18-24$ & $24(6.9)$ & $<0.001$ & $85(21.8)$ & 0.152 & $251(25.1)$ & $394(21.2)$ \\
\hline $25-29$ & $48(13.9)$ & $<0.001$ & $106(27.2)$ & 0.124 & $313(31.3)$ & $496(26.7)$ \\
\hline $30-39$ & $97(28.0)$ & 0.908 & $119(30.5)$ & 0.429 & $284(28.4)$ & $537(28.9)$ \\
\hline $40-49$ & $113(32.7)$ & $<0.001$ & $61(15.6)$ & 0.002 & $96(9.6)$ & $288(15.5)$ \\
\hline$\geq 50$ & $64(18.5)$ & $<0.001$ & $19(4.9)$ & 0.517 & $55(5.5)$ & $144(7.7)$ \\
\hline \multicolumn{7}{|l|}{ Age at first injection (yrs) } \\
\hline$<30$ & $91(26.3)$ & $<0.001$ & $228(58.5)$ & 0.005 & $671(67.2)$ & $1,065(57.3)$ \\
\hline$\geq 30$ & $255(73.7)$ & $<0.001$ & $162(41.5)$ & 0.004 & $328(32.8)$ & $794(42.7)$ \\
\hline Heterosexual condomless sex ${ }^{\dagger \dagger}$ (past $12 \operatorname{mos}^{\S \S}$ ) & $247(72.7)$ & 0.337 & $293(75.5)$ & 0.232 & $807(81.3)$ & $1,436(78.0)$ \\
\hline 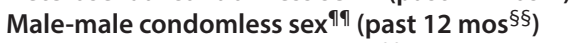 & $15(6.6)$ & 0.633 & $22(8.2)$ & 0.760 & $46(7.2)$ & $93(7.7)$ \\
\hline Shared syringe ${ }^{* * *}$ (past $12 \operatorname{mos}^{\S \S}$ ) & $96(27.8)$ & 0.006 & $123(31.6)$ & $<0.001$ & $463(46.4)$ & $734(39.5)$ \\
\hline \multicolumn{7}{|l|}{ Injection frequency (past $12 \operatorname{mos}^{\S \S}$ ) } \\
\hline$\geq 1$ time/day & $263(76.2)$ & 0.177 & $327(84.1)$ & 0.935 & $842(84.3)$ & $1,534(82.6)$ \\
\hline$<1$ time/day & $82(23.8)$ & 0.171 & $62(15.9)$ & 0.942 & $157(15.7)$ & $323(17.4)$ \\
\hline \multicolumn{7}{|l|}{ Drugs injected (past $12 \operatorname{mos}^{\S \S}$ ) } \\
\hline Heroin and other drugs ${ }^{\dagger+\dagger}$ & $164(47.4)$ & 0.092 & $229(58.7)$ & 0.037 & $659(66.2)$ & $1,132(61.0)$ \\
\hline Heroin only $\S^{\S \S}$ & $132(38.2)$ & 0.023 & $103(26.4)$ & 0.224 & $245(24.6)$ & $504(27.2)$ \\
\hline Not heroin ๆ१ฯ & $50(14.5)$ & 0.510 & $58(14.9)$ & 0.004 & $92(9.2)$ & 220 (11.9) \\
\hline \multicolumn{7}{|l|}{ Injected opioids ${ }^{* * * *}$ (past $12 \operatorname{mos}^{\S \S}$ ) } \\
\hline$\geq 1$ time/day & $6(1.7)$ & 0.239 & $19(4.9)$ & 0.228 & $37(3.7)$ & $68(3.7)$ \\
\hline$<1$ time/day & $29(8.4)$ & $<0.001$ & $62(15.9)$ & 0.010 & $228(22.8)$ & 345 (18.6) \\
\hline Never & $311(89.9)$ & $<0.001$ & $309(79.2)$ & 0.061 & $734(73.5)$ & $1,446(77.8)$ \\
\hline Injected heroin and opioids ${ }^{t+t \dagger}$ (past $12 \operatorname{mos}^{\S \S}$ ) & $32(9.3)$ & $<0.001$ & $77(19.7)$ & 0.083 & $255(25.5)$ & $390(21.0)$ \\
\hline Used noninjected opioids ${ }^{\S \S \S \S}$ (past 12 mos $^{\S \S}$ ) & $102(29.5)$ & $<0.001$ & $144(36.9)$ & 0.014 & $459(46.0)$ & $763(41.1)$ \\
\hline 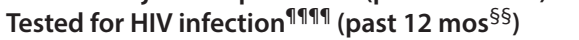 & $208(64.8)$ & 0.047 & $215(58.1)$ & 0.756 & $558(57.4)$ & $1,050(58.9)$ \\
\hline HIV prevalence ${ }^{* * * * *}$ & $29(8.6)$ & 0.004 & $15(3.9)$ & 0.187 & $20(2.0)$ & $69(3.7)$ \\
\hline Tested for HCV infection ${ }^{+t+t \dagger}$ (ever) & $232(68.6)$ & 0.239 & $244(64.0)$ & 0.021 & $697(71.6)$ & $1,263(69.6)$ \\
\hline HCV diagnosis (ever) & $45(13.4)$ & 0.003 & $71(18.8)$ & 0.539 & $211(21.7)$ & $345(19.1)$ \\
\hline Total & $346(18.6)$ & NA & $390(21.0)$ & NA & $998(53.7)$ & $1,858(100.0)$ \\
\hline
\end{tabular}

Abbreviations: $\mathrm{HCV}=$ hepatitis $\mathrm{C}$ virus; $\mathrm{HIV}=$ human immunodeficiency virus; $\mathrm{NA}=$ not applicable.

* http://www.cdc.gov/hiv/statistics/systems/nhbs/.

†The 19 cities include the following: Atlanta, Georgia; Baltimore, Maryland; Boston, Massachusetts; Chicago, Illinois; Dallas, Texas; Denver, Colorado; Detroit, Michigan; Houston, Texas; Los Angeles, California; Miami, Florida; Nassau-Suffolk, New York; New Orleans, Louisiana; New York City, New York; Newark, New Jersey; Philadelphia, Pennsylvania; San Diego, California; San Francisco, California; Seattle, Washington; Washington, D.C.

$\S$ Total column includes data on all race/ethnicity groups; therefore, percentages of blacks, Hispanics/Latinos, and whites do not total to $100 \%$.

I $p$-values calculated after adjusting for continuous age.

** Not calculated because of small sample size.

t† Condomless vaginal or anal sex with a partner of the opposite gender.

$\S \S$ In the 12 months before interview.

१ी Condomless anal sex with a male partner among male PWID.

*** Receptive syringe sharing: participant reported using a needle or syringe to inject drugs that had previously been used for drug injection by someone else.

${ }^{++\dagger}$ Injected heroin and one or more other drugs, in combination or separately, at least once. Other drugs injected include powder cocaine, crack cocaine, methamphetamine, and prescription painkillers.

$\S \S \S$ Injected heroin, but did not report injecting any other drug.

१ศๆ Injected one or more drugs, but not heroin.

**** Injected prescription painkillers such as Oxycontin, Dilaudid, morphine, Percocet, or Demerol; does not include injection of heroin.

$\mathrm{tt+t}^{+}$Injected heroin and prescription painkillers, such as Oxycontin, Dilaudid, morphine, Percocet, or Demerol, in combination or separately, at least once.

$\S \S \S \S$ Noninjection of prescription painkillers such as Oxycontin, Dilaudid, morphine, Percocet, or Demerol; does not include heroin.

กศศๆ Among participants who did not report a previous HIV-positive test result.

***** Had a confirmed HIV-positive test result during interview.

ttttt Self-reported, lifetime diagnosis of HCV infection among participants who reported having ever been tested for HCV infection. 
The most effective way for persons to avoid acquiring HIV and other negative health outcomes associated with injection drug use, including HCV, hepatitis B virus, abscesses, bacterial endocarditis, skin and soft tissue infections, and overdose, is not to inject drugs. For those who do inject, provision of sterile syringes and services through SSPs can decrease risk for HIV transmission and other negative health outcomes. A need to address injection drug use and associated risk behaviors exists because of several factors, including recent increases in heroin addiction and overdose ( 6 ); the HIV outbreak in Scott County, Indiana, which saw an increase in diagnoses from five in 5 years to 181 in 1 year (14); and the recent $364 \%$ increase of $\mathrm{HCV}$ transmission in rural areas (16), largely fueled by the current U.S. opioid epidemic (11). The window of opportunity for implementing SSPs that provide comprehensive services to prevent, rather than respond to, HIV outbreaks might be closing. Swift action can lead to further decreases in HIV diagnoses and prevent new outbreaks among PWID.

\section{Acknowledgments}

Laura Eastham, Dita Broz.

${ }^{1}$ National Center for HIV/AIDS, Viral Hepatitis, STD, and TB Prevention, CDC.

Corresponding author: Cyprian Wejnert, cwejnert@cdc.gov, 404-639-6055.

\section{References}

1. CDC. HIV surveillance among persons who inject drugs [slide set]. Atlanta, GA: US Department of Health and Human Services; 2016. http://www.cdc.gov/hiv/library/slidesets/index.html

2. Oster AM, Sternberg M, Lansky A, Broz D, Wejnert C, Paz-Bailey G. Population size estimates for men who have sex with men and persons who inject drugs. J Urban Health 2015;92:733-43. http://dx.doi. org/10.1007/s11524-015-9970-3

3. CDC. Diagnoses of HIV infection in the United States and dependent areas, 2015. HIV Surveillance Report. Vol. 27. Atlanta, GA: US Department of Health and Human Services, CDC; 2016. https://www.cdc.gov/hiv/pdf/ library/reports/surveillance/cdc-hiv-surveillance-report-2015-vol-27.pdf

4. CDC. Viral Hepatitis Surveillance-United States, 2014. Atlanta, GA: US Department of Health and Human Services, CDC; 2016. https://www.cdc. gov/hepatitis/statistics/2014surveillance/pdfs/2014hepsurveillancerpt.pdf
5. CDC. Vital signs: overdoses of prescription opioid pain relievers and other drugs among women—United States, 1999-2010. MMWR Morb Mortal Wkly Rep 2013;62:537-42.

6. Jones CM, Logan J, Gladden RM, Bohm MK. Vital signs: demographic and substance use trends among heroin users-United States, 20022013. MMWR Morb Mortal Wkly Rep 2015;64:719-25.

7. Harrison KM, Kajese T, Hall HI, Song R. Risk factor redistribution of the national HIV/AIDS surveillance data: an alternative approach. Public Health Rep 2008;123:618-27.

8. Lansky A, Abdul-Quader AS, Cribbin M, et al. Developing an HIV behavioral surveillance system for injecting drug users: the National HIV Behavioral Surveillance System. Public Health Rep 2007;122(Suppl 1):48-55.

9. Des Jarlais DC, Nugent A, Solberg A, Feelemyer J, Mermin J, Holtzman D. Syringe service programs for persons who inject drugs in urban, suburban, and rural areas-United States, 2013. MMWR Morb Mortal Wkly Rep 2015;64:1337-41. http://dx.doi.org/10.15585/mmwr.mm6448a3

10. Broz D, Ouellet LJ. Racial and ethnic changes in heroin injection in the United States: implications for the HIV/AIDS epidemic. Drug Alcohol Depend 2008;94:221-33. http://dx.doi.org/10.1016/j. drugalcdep.2007.11.020

11. Cicero TJ, Ellis MS, Surratt HL, Kurtz SP. The changing face of heroin use in the United States: a retrospective analysis of the past 50 years. JAMA Psychiatry 2014;71:821-6. http://dx.doi.org/10.1001/ jamapsychiatry.2014.366

12. Broz D, Pham H, Spiller M, et al. Prevalence of HIV infection and risk behaviors among younger and older injecting drug users in the United States, 2009. AIDS Behav 2014;18(Suppl 3):284-96. http://dx.doi. org/10.1007/s10461-013-0660-4

13. Van Handel MM, Rose CE, Hallisey EJ, et al. County-level vulnerability assessment for rapid dissemination of HIV or HCV infections among persons who inject drugs, United States. J Acquir Immune Defic Syndr 2016;73:323-31. http://dx.doi.org/10.1097/QAI.0000000000001098

14. Peters PJ, Pontones P, Hoover KW, et al.; Indiana HIV Outbreak Investigation Team. HIV infection linked to injection use of oxymorphone in Indiana, 2014-2015. N Engl J Med 2016;375:229-39. http://dx.doi.org/10.1056/NEJMoa1515195

15. Bramson H, Des Jarlais DC, Arasteh K, et al. State laws, syringe exchange, and HIV among persons who inject drugs in the United States: history and effectiveness. J Public Health Policy 2015;36:212-30. http://dx.doi. org/10.1057/jphp.2014.54

16. Zibbell JE, Iqbal K, Patel RC, et al. Increases in hepatitis $C$ virus infection related to injection drug use among persons aged $\leq 30$ years-Kentucky, Tennessee, Virginia, and West Virginia, 2006-2012. MMWR Morb Mortal Wkly Rep 2015;64:453-8. 